\title{
A Single Impurity in Tomonaga-Luttinger Liquids.
}

\author{
Yuval Oreg and Alexander M. Finkel'stein \\ Department of Condensed Matter Physics, The Weizmann \\ Institute of Science, \\ Rehovot 76100, Israel
}

(March 1997)

\begin{abstract}
The problem of a single impurity in one dimensional Tomonaga -Luttinger liquids with a repulsive electron-electron interaction is discussed. We find that the renormalization group flow diagram for the parameters characterizing the impurity is rather complex. Apart from the fixed points corresponding to two weakly connected semi-infinite wires, the flow diagram contains additional fixed points which control the low temperature physics when the bare potential of the impurity is not strong.
\end{abstract}

\section{INTRODUCTION}

The recent advance in submicron technology enables fabrication of truly one-dimensional (1D) quantum wires. The electron liquids in these systems are usually described in terms of the Tomonaga-Luttinger (TL) model [1.2]. Edge states in a two-dimensional electron gas, under conditions of the fractional quantum Hall effect, were argued to be TL liquids as well [3]. It is well known that in the TL model with a repulsive electron-electron interaction the effective strength of a backward scattering by an impurity defect increases with decreasing temperature [4. For this reason, the conductance of TL liquids with a single defect has been 
intensively discussed recently using various theoretical methods [5 9. It has been concluded that even a weak impurity eventually causes the conductance to vanish at low temperatures.

The physical interpretation of the conductance vanishing is based on the assumption made by Kane and Fisher [10], that in the limit of low temperatures the behavior of a TL system with an impurity may be described as tunneling between two disconnected semiinfinite TL wires. The effective amplitude of tunneling between the half-wires scales to zero with decreasing temperature, because the tunneling density of states at the ending point of a TL liquid vanishes when the electron-electron interaction is repulsive [10,11]. This interpretation corresponds to a scenario in which the effective strength of the impurity increases in the course of the renormalization, so that at the final stage a weak impurity transforms into a strong barrier, and disconnects the TL wire. However, a direct calculation of the tunneling density of states [12, obtained by a mapping of the weak impurity problem onto a Coulomb gas theory, apparently contradicts this intuitive picture. It has been found that at the location of a weak impurity the tunneling density of states is enhanced, rather than vanishing. The scenario of Ref. [10] is based on the assumption that no other fixed points intervene in the scaling from the repulsive fixed point of a weakly scattering defect to the attractive fixed point corresponding to a tunneling junction of two half-wires. The contradiction of this scenario to the calculations of single particle properties, such as the tunneling density of states, and the Fermi edge singularity [13], indicates that maybe this is not the case.

In this work the problem of a single impurity in TL liquids with a repulsive electronelectron interaction is reinvestigated. We concentrate on the limit when the Fermi wave length is much larger than the defect size. This situation is typical for semiconductors, where the filling of the conduction band is far from one half. Then it is possible to describe the problem as a continuous model with an appropriately chosen point-like defect. We come to the conclusion that the low energy physics of a weak impurity is controlled by a fixed point that differs from the one corresponding to a tunneling junction of two half-wires. This aspect of the discussed problem proves to be similar to an overscreened two channel 
Kondo problem, while the original idea of the theory of Ref. [10] has been orientated to a situation similar to the ordinary Kondo problem (see also Ref. [14 for a discussion). The above statement may seem to be strange. Indeed, in the overscreened two channel Kondo problem the infinitely strong exchange interaction corresponds to a repulsive fixed point [15], while in the problem under discussion the only apparent candidate for a fixed point in the strong coupling regime is the fixed point of a tunneling junction [16], which is attractive. We find here that there exists another fixed point corresponding to the infinitely strong amplitude of the impurity backward scattering, and it is repulsive. This implies that in addition there should be an attractive fixed point at a finite value of the backward scattering amplitude. The renormalization group (RG) flow diagram of the problem depends on several parameters characterizing the impurity, and is rather complex. Each of the fixed points describing the backward scattering problem, as well as that of the tunneling junction, corresponds to a manifold of fixed points. An important fact is, that the tunneling junction manifold, and the two of the new fixed points describing backward scattering, are located in different parts of the RG flow diagram.

For a point-like defect, all Fourier components of an impurity potential are practically the same. Therefore, one may characterize the "strength" of the potential by an initial (bare) value of a dimensionless parameter $u_{+}=\int U(x) d x / v_{F}$, where $v_{F}$ is the Fermi velocity. When $u_{+}$is small, the backward scattering problem is described by the partition function $Z_{s c}\left(u_{-} ; g\right)$, depending on a backward scattering amplitude $u_{-}$, and on the electron-electron interaction parameter $g$. Alternatively, when $u_{+}$is large, i.e., the impurity is strong, the appropriate description is a tunneling model with a partition function $Z_{t u n}\left(t_{-} ; g\right)$, depending on a tunneling amplitude $t_{-}$. For free electrons, the two partition functions are deeply connected, because of the relation between the reflection from and the transmission through the defect. In this case, in principle, each one of them can represent an impurity of an arbitrary strength. The situation becomes quite different when the electron-electron interaction is switched on in TL liquids. Then, the partition functions $Z_{s c}\left(u_{-} ; g\right)$ and $Z_{\text {tun }}\left(t_{-} ; g\right)$ are not equivalent anymore. Instead, they describe two complementary limiting cases of the phase 
diagram, and give completely different structures of the RG fixed points for these cases.

In this paper, the RG flow diagram of the continuous model is analyzed by examining different "corners" of the scattering and the tunneling limits, and tailoring them together. The flow diagram is constructed in axes representing the strength of the impurity $u_{+}$, and the backward scattering amplitude $u_{-}$(and/or $\left.1 / t_{-}\right)$, respectively. The presentation of the phase diagram in the space of the impurity potential parameters, rather than the conventional presentation in terms of the backward scattering amplitude together with the parameter $g$, helps clarify the different character of the fixed points controlling the low energy physics in the cases of a weak and a strong bare impurity potential. In the course of renormalization of a weak impurity the effective barrier evolves in a special manner. Only the backward scattering amplitude $u_{-}$increases, while $u_{+}$, being marginal, remains small. That is why a weak local impurity in a continuous model does not evolve towards a strong barrier, but proceeds to scale in the other, complementary, part of the RG flow diagram. The low energy physics in this case is controlled by a line of fixed points $L_{f}$, at a finite value of $u_{-}$. (More precisely, $L_{f}$ is a manifold of fixed points due to the presence of other marginal parameters. Since we use a two-dimensional plot for the RG-diagram, we will call such manifolds "lines".) The other part of the flow diagram contains a line of attractive fixed points, $D_{0}$, controlling the low energy physics when the bare potential of the impurity is strong. This line describes the vanishing of the tunneling amplitude $t_{-}$, and corresponds to two disconnected half-wires.

We think that the attraction of the weak impurity problem to $L_{f}$, but not to $D_{0}$, is the reason for the difference between the results obtained by means of the Coulomb gas theory for the single particle correlators, and those obtained relying on the scenario of two disconnected wires.

The paper is organized as follows: in Sec. II we discuss the renormalization of the scattering and the tunneling Hamiltonians, i.e., the RG flow for two different limits of the impurity strength. The existence of several parameters describing a local defect in the continuous model is emphasized. In Sec. III a RG flow diagram unifying both cases of a weak and of a strong impurity potential is discussed. In Appendix A the repulsive character of the 
limit $u_{-}=\infty$ and, consequently, the existence of the attractive $L_{f}$-line, is demonstrated by mapping the impurity problem onto a spin-1/2 Heisenberg chain.

\section{THE REGIONS OF A WEAK AND A STRONG IMPURITY IN THE FLOW DIAGRAM}

In this section we reproduce the main results concerning the renormalization of the problem in the limits of a weak and of a strong impurity potential. We assume that the Fermi wave length is much larger than the defect size. This situation occurs in semiconductor wires where the filling of the conduction band is far from one half and the Fermi wave length is large. Several authors used the half-filled tight binding model with a link defect, to analyze the problem of backward scattering in quantum wires. The tight binding model corresponds to a fixed choice of the parameters describing the defect, that makes this model a specific one. In this case the Fermi wave length is commensurate with the impurity size, and the final fixed point depends on the internal structure of the defect [14]. The continuous model considered here is not constrained by the specific structure of the local defect.

\section{A weak potential scattering: the repulsive line $L_{0}$}

The Hamiltonian of the TL model is given by

$$
H_{T L}=-i v_{F} \int d x \psi_{R}^{\dagger}(x) \frac{\partial}{\partial x} \psi_{R}(x)+i v_{F} \int d x \psi_{L}^{\dagger}(x) \frac{\partial}{\partial x} \psi_{L}(x)+\frac{1}{2} V \int d x\left(\rho_{R}(x)+\rho_{L}(x)\right)^{2},
$$

Here the electron spectrum is linearized near the Fermi points $\pm k_{F} ; \psi_{R}(x)$ and $\psi_{R}^{\dagger}(x)$ are the field operators of fermions that propagate to the right with wave vectors $\approx+k_{F}$, and $\psi_{L}(x)$ and $\psi_{L}^{\dagger}(x)$ are the field operators of left propagating fermions with wave vectors

$\approx-k_{F} ; \quad \rho_{L(R)}(x)=\psi_{L(R)}^{\dagger}(x) \psi_{L(R)}(x)$ are the electron density operators; $V$ describes the density-density interaction with a momentum transfer much smaller than $k_{F}$. The Hamiltonian (1) describes the 1D electron liquid when the backward scattering amplitude of the 
electron-electron interaction may be ignored, and it is a fixed point Hamiltonian for a broad class of $1 \mathrm{D}$ systems.

For low energy physics only processes of electron scattering with a momentum transfer close to zero and to $2 k_{F}$ are essential. Let us denote

$$
U_{0}=\int d x U(x), \quad U_{2 k_{F}}=\int d x U(x) e^{i 2 k_{F} x}
$$

Here $U_{0}$ and $U_{2 k_{F}}=-\left|U_{2 k_{F}}\right| e^{i \varphi_{u}}$ are the Fourier transform amplitudes of the impurity potential $U(x)$. For a weak impurity the forward and backward scattering amplitudes are

$$
u_{+}=U_{0} / v_{F}, \quad u_{-}=\left|U_{2 k_{F}}\right| / v_{F},
$$

respectively; $v_{F}$ is the Fermi velocity. (To avoid confusion, we will not use here the notations $\delta_{ \pm}$for the dimensionless amplitudes $u_{ \pm}$, unlike in [13], because the relations of the amplitudes $u_{ \pm}$with the scattering phase shifts are ill defined for interacting electrons in the TL model.) Note, that since the bare impurity potential $U(x)$ is assumed to be local, it has Fourier components of the same order for practically all momenta. Therefore, the line of the bare parameters corresponds to $u_{+} \approx u_{-}$.

In addition to $u_{+}, u_{-}$, and $\varphi_{u}$, another parameter, $u_{a}$, describing the asymmetry of the forward scattering of left and right movers will be introduced. In the presence of time reversal symmetry $u_{a} \equiv 0$, but it is not necessarily zero for the quantum Hall edge states. The scattering of the conduction band electrons by a single local defect at $x=0$ is given by

$$
\begin{aligned}
H_{s c}= & v_{F}\left[u_{+}\left(\psi_{R}^{\dagger}(0) \psi_{R}(0)+\psi_{L}^{\dagger}(0) \psi_{L}(0)\right)+u_{a}\left(\psi_{R}^{\dagger}(0) \psi_{R}(0)-\psi_{L}^{\dagger}(0) \psi_{L}(0)\right)\right. \\
& \left.-u_{-}\left(e^{i \varphi_{u}} \psi_{R}^{\dagger}(0) \psi_{L}(0)+e^{-i \varphi_{u}} \psi_{L}^{\dagger}(0) \psi_{R}(0)\right)\right]
\end{aligned}
$$

where $\psi_{R(L)}(0) \equiv \psi_{R(L)}(x=0)$.

The bosonization technique (for review see [1,2]) allows one to reduce the TL Hamiltonian to a quadratic form in terms of operators of bosonic fields $\phi_{0}$ and $\tilde{\phi}_{0}$ :

$$
\begin{aligned}
& \phi_{0}(x)=\frac{-i}{\sqrt{4 \pi}} \frac{2 \pi}{L} \sum_{p} \frac{\exp (-\eta|p| / 2-i p x)}{p}\left[\rho_{R}(p)+\rho_{L}(p)\right], \\
& \tilde{\phi}_{0}(x)=\frac{-i}{\sqrt{4 \pi}} \frac{2 \pi}{L} \sum_{p} \frac{\exp (-\eta|p| / 2-i p x)}{p}\left[\rho_{R}(p)-\rho_{L}(p)\right],
\end{aligned}
$$


where $L$ is the system length, and $\eta^{-1}$ is an ultraviolet cutoff, $(2 \pi \eta)^{-1}$ is equal to the average density of the electron liquid. The fields $\phi_{0}$ and its dual partner $\tilde{\phi}_{0}$ are conjugate variables, i.e.,

$$
\left[\frac{d \phi_{0}(x)}{d x}, \tilde{\phi}_{0}(y)\right]=i \delta(x-y)
$$

After rescaling the operators

$$
\phi=\frac{\sqrt{4 \pi}}{\beta} \phi_{0}, \tilde{\phi}=\frac{\beta}{\sqrt{4 \pi}} \tilde{\phi}_{0},
$$

the bosonized representation of the Hamiltonian (II) becomes

$$
H_{T L}^{B}=\frac{v_{F}}{2 g} \int d x\left(\left(\frac{d \phi}{d x}\right)^{2}+\left(\frac{d \tilde{\phi}}{d x}\right)^{2}\right)
$$

Here $g$ is an effective parameter of the electron-electron interaction

$$
g=\frac{\beta^{2}}{4 \pi}, \beta^{2}=4 \pi \sqrt{\frac{1-\gamma}{1+\gamma}}, \quad \gamma=\frac{V}{\left(2 \pi v_{F}+V\right)}
$$

$g<1$ when the electron-electron interaction is repulsive, while for the attractive interaction $g>1$.

The bosonic representations of the operators $\psi_{R}$ and $\psi_{L}$ are given as

$$
\begin{aligned}
& \psi_{R}^{B}(x)=\frac{e^{i k_{F} x}}{\sqrt{2 \pi \eta}} \exp \left[-\frac{i}{2}\left(\frac{4 \pi}{\beta} \tilde{\phi}+\beta \phi\right)\right], \\
& \psi_{L}^{B}(x)=i \frac{e^{-i k_{F} x}}{\sqrt{2 \pi \eta}} \exp \left[-\frac{i}{2}\left(\frac{4 \pi}{\beta} \tilde{\phi}-\beta \phi\right)\right] .
\end{aligned}
$$

Then, the scattering by a weak impurity may be written in terms of the $\phi$-field as

$$
H_{s c}^{B}=\frac{v_{F}}{2 \pi}\left(\left.\beta u_{+} \frac{d \phi}{d x}\right|_{x=0}+\left.\frac{4 \pi}{\beta} u_{a} \frac{d \tilde{\phi}}{d x}\right|_{x=0}-\frac{2 u_{-}}{\eta} \cos \left(\beta \phi(0)+\varphi_{u}\right)\right) .
$$

The RG equations for this problem are

$$
\begin{gathered}
\frac{d u_{-}}{d \xi}=u_{-}(1-g), \\
\frac{d u_{+}}{d \xi}=0,
\end{gathered}
$$




$$
\begin{gathered}
\frac{d u_{a}}{d \xi}=0, \quad \frac{d \varphi_{u}}{d \xi}=0, \\
\frac{d g}{d \xi}=0 .
\end{gathered}
$$

The last equation here reflects the well known fact that in the TL model the interaction parameter $g$ is not renormalized. Since $g$ describes a 'bulk' electron liquid, the presence of a single impurity cannot influence this parameter. The decoupling of the RG-equations for amplitudes $u_{+}$from the rest of the parameters is a consequence of an important property of the Hamiltonian $H_{T L}+H_{s c}$. Namely, it can be split into two commuting parts containing the forward $u_{+}$, and the backward $u_{-}$, scattering terms separately [17,18], see Eq. (A3). Since the forward scattering $u_{+}$is given by a linear term in Eq. (13), and the amplitude $u_{-}$cannot influence the renormalization of $u_{+}$because of the separation, the fulfillment of Eq. (14b) becomes evident. Hence, under the condition that the impurity can be described as a local weak potential in a TL liquid, the forward scattering amplitude $u_{+}$is a marginal parameter. The parameters $u_{a}$ and $\varphi_{u}$ are marginal as well, see Eq. (14d). This is because the $u_{a}$-term, being linear in $d \tilde{\phi} / d x$, can be removed from the Hamiltonian by a canonical transformation, while $\varphi_{u}$ does not reveal itself in the partition function $Z_{s c}$. In contrast, the backward scattering amplitude is relevant for the repulsive case. Eq. (14a) has been derived [5] (see also [19]) for small $u_{-}$. The renormalization of $u_{-}$in the strong coupling regime is discussed in Sec. [II].

Eqs. (14a 14d) describe a repulsive manifold of fixed points $L_{0}$, denoted as the $L_{0}$-line at the left-bottom corner, $u_{ \pm} \ll 1$, of the RG-plane depicted in Fig. 1. 


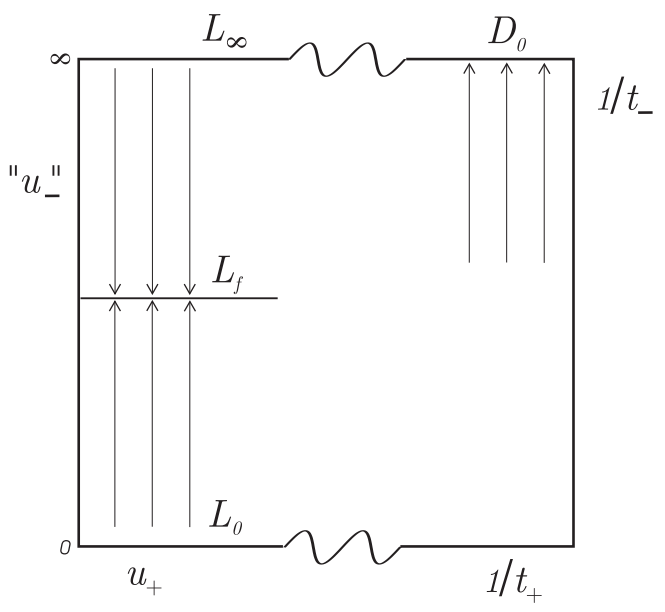

FIG. 1. The RG-flow diagram of a point-like defect in a quantum wire with a repulsive electron-electron interaction: The line $L_{0}$ represents a manifold of fixed points in the case of an infinitesimally small impurity potential. The line $D_{0}$ corresponds to the tunneling junction limit. The line $L_{\infty}$ corresponds to the limit of an infinitely large backward scattering amplitude $u_{-}$in Hamiltonian (13). The attractive fixed line $L_{f}$ controls the low temperature physics of the backward scattering problem. The problems of a weak impurity and a weak tunneling junction evolve at complementary parts of the phase diagram, and do not flow to each other in the model with a linearized electron spectrum.

\section{A strong potential barrier: the line of attractive fixed points $D_{0}$}

When the bare impurity is strong enough, the description of the problem in terms of the scattering amplitudes $u_{-}, u_{+}, u_{a}$, and $\varphi_{u}$ ceases to be adequate. On the other hand, the fact that the impurity is strong does not contradict the assumption of locality $k_{F} a \ll 1$. A local and strong impurity can also be considered as a point-like problem, but here it should be described by two semi-infinite TL liquids, with a weak tunneling junction between their ending points. Like in the case of the weak potential scattering, there are four parameters that describe the tunneling and reflection processes at the tunneling junction of the two half-wires. These parameters are the tunneling amplitude $t_{-}$, its phase $\varphi_{t}$, and the two parameters, $t_{+}$and $t_{a}$, characterizing the phases that an electron acquires when it is reflected at the ends of the half-wires. The parameter $t_{a}$ describes the asymmetry of the left and the 
right parts of the tunneling junction. In the particular case of a strong $\delta$-function potential, where $u_{ \pm}=u \gg 1$, the amplitudes $t_{ \pm} \sim 1 / u$.

The low energy physics of each semi-infinite wire may be described by a single chiral mode [5. 18]. It will be assumed that there is no density-density interaction between the half-wires, but inside each of them the density-density interaction is present. The effects of the electron-electron interaction inside a half-wire can be taken into consideration by a canonical transformation (see ,e.g., Sec. IIIA in Ref. [13]). Then the appropriate bosonic description of tunneling between two oppositely moving chiral modes corresponding to each of the half-wires is given by

$$
H_{t}^{B}=\frac{v_{F}}{2 \pi}\left(\left.\frac{4 \pi}{\beta} t_{+} \frac{d \phi_{\text {tun }}}{d x}\right|_{x=0}+\left.\beta t_{a} \frac{d \tilde{\phi}_{\text {tun }}}{d x}\right|_{x=0}-\frac{2 t_{-}}{\eta} \cos \left(\frac{4 \pi}{\beta} \phi_{\text {tun }}(0)+\varphi_{t}\right)\right) .
$$

Here $d \phi_{t u n}(x) / d x$ is related to the total density of the two chiral modes, and $\tilde{\phi}_{t u n}$ is a field dual to $\phi_{t u n}$.

The RG equations in this case are analogous to Eqs. (14ar-14d):

$$
\begin{gathered}
\frac{d t_{-}}{d \xi}=t_{-}(1-1 / g), \\
\frac{d t_{+}}{d \xi}=0, \\
\frac{d t_{a}}{d \xi}=0, \quad \frac{d \varphi_{t}}{d \xi}=0 .
\end{gathered}
$$

In contrast to $u_{-}$, the tunneling amplitude $t_{-}$scales to zero for repulsive electron-electron interaction $(g<1)$. Therefore, the manifold of fixed points described by the tunneling Hamiltonian $H_{t}^{B}$ is attractive. In the two-dimensional plot depicted in Fig. 1 it is presented in the upper right corner as the $D_{0}$-line.

\section{THE UNIFIED RG FLOW DIAGRAM}

The problem of an impurity in a TL liquid has been discussed in Sec. II for the two limiting models: an impurity scattering, Eq.(13), and a tunneling junction, Eq.(15)). The 
scaling flow diagrams of these complementary models are depicted together in Fig. 11 as a combined plot. In this plot, the scattering model is represented on the left side, and the tunneling model - on the right one. The center of the plot corresponds to the crossover region when the impurity potential has an intermediate strength. Since for a large barrier the backscattering amplitude is large, and the tunneling amplitude is small, we use the vertical axis to represent $u_{-}$and $1 / t_{-}$. The horizontal axis represents $u_{+}$together with the other parameters, which in a model with a linearized electron spectrum are not renormalized. Due to the presence of these additional parameters, lines on the symbolic two-dimensional plot correspond to hyperplanes.

For a point-like impurity there are certain relations between the bare parameters characterizing the potential: $u_{-} \approx u_{+}$, and $1 / t_{-} \approx 1 / t_{+}$. Hence, only the left-bottom and the right-upper corners of the phase diagram in Fig. 1 are attainable for bare parameters of a local impurity in the continuous model. However, according to Eqs. (14a), (14b) the amplitude $u_{-}$renormalizes to a strong coupling regime, while $u_{+}$being marginal, remains small. Therefore, to understand the nature of this regime, we have to analyze the scattering Hamiltonian (13) for the region of the parameters corresponding to the upper left corner of the phase diagram. (Since large $u_{-}$together with small $u_{+}$does not correspond to a physical realization of the bare parameters, we use in the phase diagram " $u_{-}$" with quotation marks.) In Appendix $\mathrm{A}$, the line $L_{\infty}$ with $u_{-} \gg 1$ and a not too large $u_{+}$is argued to be a repulsive fixed line. For this purpose, the Hamiltonian $H=H_{T L}+H_{s c}$ of the impurity scattering in the TL model is mapped onto a semi-infinite spin- $1 / 2$ Heisenberg chain. In this mapping the impurity backscattering amplitude corresponds to a magnetic field $h \propto u_{-}$, that acts on the spin located at the origin of the chain. In the course of the RG treatment small $h$ scales, as $u_{-}$does, to a strong coupling regime. To investigate the nature of this regime, we analyze the stability of the limit $h=\infty$, following the spirit of the treatment of the two channel Kondo problem by Nozières and Blandin [15]. When $h$ is very large, the spin-spin coupling in the spin chain can be considered as a small perturbation in a real space RG analysis. This procedure generates a new operator, which is not irrelevant. 
If one assumes that $h \rightarrow \infty$ is a stable fixed point, the latter fact makes the RG process nonconvergent. This is in contradiction to the assumption of stability, and therefore we have to conclude that $h \rightarrow \infty$ is a repulsive fixed point. Since $h \propto u_{-}$, we get that the limit of an infinitely strong backward scattering is repulsive. Both limits, $u_{-}=0$ and $u_{-}=\infty$, are repulsive, and therefore there necessarily should be an attractive fixed point at some finite value of the backward scattering amplitude $u_{-}$. (This analysis is valid when a description of the local impurity scattering in terms of the TL model is a good approximation. Then the Hamiltonian $H$ can be split into two commuting parts describing the backward and the forward scattering separately [17,18], see Eq. (A3). The discussed RG-regime of the $u_{-}$-amplitude develops inside the backward scattering part alone.) Thus, on the left side of the diagram there are two repulsive lines $L_{0}$ and $L_{\infty}$, and in addition an attractive line $L_{f}$, which is sandwiched between the opposite directions of the flow. The right side of the diagram corresponding to the tunneling Hamiltonian (15), contains an attractive fixed line $D_{0}$ which describes scaling to zero of the tunneling amplitude $t_{-}$.

The information collected up to now is presented on the combined plot in Fig. 11. To complete the central part of the flow diagram, a region of an intermediate impurity strength should be studied. None of the two limiting models describes the problem faithfully in this crossover region, and a consideration of a more comprehensive Hamiltonian, which covers both limiting cases, is needed. Moreover, to study the RG-flow in the crossover region, one has to give up the approximations of the linearized electron spectrum and/or of the locality of the defect. Then, the decoupling of forward and backward scattering is no longer valid. (In the bosonized representation terms $\propto \rho_{L(R)}^{3}$ describe the curvature of the electron spectrum. One of the possible ways to consider the nonlocality of the impurity is to add a term $\propto \psi_{R}^{\dagger}(0) \frac{d}{d x} \psi_{L}(0)+h . c$. . When $k_{F} a \ll 1$ the coefficient of this term is very small.) Effects arising due to the nonlinearity of the electron spectrum and the nonlocality of the defect should be studied by a loop expansion in higher orders. These effects may have highly important influence on the renormalization of the parameters $u_{+}$and $u_{-}$. For example, it may cause the flow lines near the $L_{f}$-line to bend to the left or to the right. 
The plot in Fig. 1 is based on the idealized models, and as a draft it gives a hint how the known limiting cases could be matched together. Since the curvature of the electron spectrum is not universal, different scenarios can occur. The two most apparent versions of the flow diagram are presented in Figs. $2 a$ and $2 b$, but more sophisticated variants can be imagined due to multidimensionality of the problem, which up to now was hidden by the linearized spectrum approximation. In the version presented in Fig. 2a $a$, the limiting cases of a weak and a strong impurity evolve completely independently. In contrast, the RG-flow presented in Fig. $2 b$ corresponds to a scaling from $L_{0}$ to $D_{0}$, i.e., from a weak impurity scattering to a limit of two disconnected half-wires, as it has been assumed in Ref. [10]. However, this version of the RG-flow acquires, in the present discussion, a new essential element. Namely, the flow trajectory after the first stage where it reaches the $L_{f}$-line dwells at length in its vicinity, and this leads to an intermediate asymptotic behavior. For a weak enough impurity this intermediate regime can be very long, and then it determines the low energy physics in a certain temperature range.
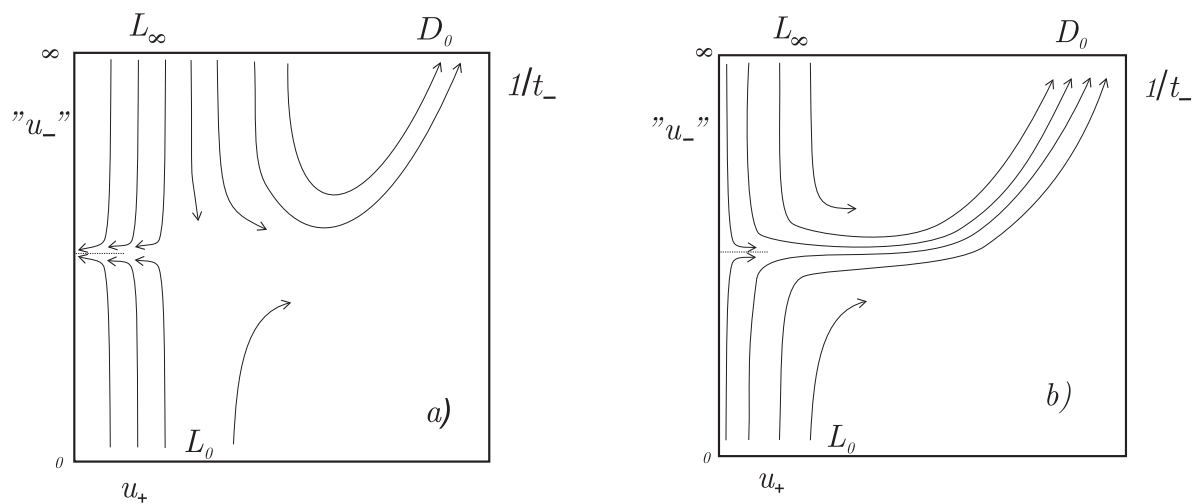

FIG. 2. Two possible modifications of the flow diagram that can occur beyond the approximations of the model, such as the linearization of the spectrum, the locality of the defect, etc. The flow diagram becomes dependent on the model parameters in a non universal way.

We emphasize that in considerations based on the approximation of a linearized electron spectrum, RG-trajectories that start at $L_{0}$ end at $L_{f}$. This approximation has been utilized in the mapping of the problem onto a Coulomb gas theory [13,12]. Therefore, the tunneling density of states, and the Fermi edge singularity exponent, found in Refs. [13, 12] correspond 
to the physics near $L_{f}$, and not to a tunneling junction, i.e., not near $D_{0}$.

\section{SUMMARY}

We have studied a single impurity in TL liquids with a repulsive electron-electron interaction. The problem has been described by a continuous model with a point-like defect. Two complementary limiting models corresponding to the weak and the strong impurity potential limits have been presented on a unified flow diagram depicted in Fig. 1. Apart from the backward scattering amplitude for a weak impurity, and the tunneling amplitude at the opposite extreme, another parameter characterizing the "strength" of the impurity potential controls the RG-flow. The unified plot of the flow diagram is rather complex. Because of many marginal parameters, fixed points of the limiting problems generate manifolds which on the symbolic two-dimensional plot of the flow diagram we present as lines. When the potential barrier of the impurity is strong, the low temperature behavior is controlled by the $D_{0}$-line corresponding to two disconnected semi-infinite wires. In addition, the flow diagram contains another attractive fixed line, $L_{f}$, controlling the low temperature physics when the bare potential of the impurity is weak. The existence of this line has been established by considering a scattering Hamiltonian (13) with the backward scattering parameter taken to be very strong, $u_{-} \rightarrow \infty$, while the forward one, $u_{+}$, was small. This somewhat fictitious case is important due to the fact that in the TL model only the backward scattering scales to strong coupling while $u_{+}$remains marginal. By mapping this case onto a spin- $1 / 2$ Heisenberg chain, it has been shown that the line $L_{\infty}$ describing the limit $u_{-} \rightarrow \infty$ is unstable. The existence of the attractive line $L_{f}$ at an intermediate value of the parameter $u_{-}$, follows from the fact that both limiting lines $L_{\infty}$ and $L_{0}$, have proved to be unstable.

The renormalization of the $u_{-}$-amplitudes resembles the situation of the overscreened two channel Kondo problem [15]. This is not accidental. At a particular value of the electronelectron interaction parameter $g=1 / 2$, the problem of a weak impurity in a TL liquid can be mapped onto the two channel Kondo model with a specific value of the longitudinal exchange 
coupling (for details see the end of Appendix A). It is well known that in the overscreened two channel Kondo problem the limit of infinite exchange interaction is unstable, and there is an anomalous fixed point at a finite coupling. Since the problem under consideration, and the overscreened two channel Kondo model are equivalent at one point, it is natural that the line $u_{-}=\infty$ proves to be repulsive, as it has been found here. Note, however, that the present treatment is not restricted to a specific value of the electron-electron interaction.

The presentation of the phase diagram in the space of parameters characterizing the impurity potential, helps clarify the difference between the $D_{0^{-}}$and the $L_{f^{-}}$lines of fixed points-they are located in different parts of the phase diagram. In the continuous model with a linearized electron spectrum the two limiting cases of a point-like defect's strength evolve completely independently. This is constructive for understanding the low temperature physics in the case of a weak impurity. On the other hand, an extension beyond the assumption of the model such as the linearization of the spectrum, the locality of the defect, as well as other possible mechanisms of a crossover between different regimes, remain open questions.

A novel line of fixed points can be essential for the scenario of Ref. [10]. This scenario is based on the assumption of scaling from a weak impurity scattering to a strong barrier. The existence of the $L_{f}$-line indicates that the situation is more complicated (see the discussion of Figs. 2 $2 a, 2 b$ at the end of Sec. III). We think that the attraction of the weak impurity problem to $L_{f}$, but not to $D_{0}$, is the reason for the results obtained by means of the Coulomb gas theory [12,13]. The enhancement of the tunneling density of states obtained in this theory corresponds to decrease of the escape rate of an electron from a defect center, as a result of multiple backward scattering in combination with the electron-electron interaction. We believe that the physics of the $L_{f}$-line may have a relation to the strengthening of the role of the Friedel oscillations in the TL model, see e.g., Ref. [6].

To conclude, we have developed a continuous model of a single local defect in a TL liquid. This theory is related to the physics of semiconducting $1 D$ quantum wires, and edge states in the quantum Hall effect. We identify a new attractive fixed point controlling the strong 
coupling regime of the backward scattering in the TL model. This novel point may also have implications for some other related problems, in particular to the theory of the motion of a quantum particle in a dissipative environment, see Ref. 20] and references therein.

\section{ACKNOWLEDGMENTS}

We thank Y. Gefen and D. E. Khmelnitskii for useful discussions. A. F. is supported by the Barecha Fund Award. The work is supported by the Israel Academy of Science under the Grant No. 801/94-1 and by German-Israel Foundation (GIF).

\section{APPENDIX A:}

In this Appendix the problem of a local impurity in the TL model is mapped onto a semiinfinite spin-1/2 Heisenberg chain, with a magnetic field $h \propto u_{-}$acting on the spin located at origin of the chain. The mapping onto a spin chain is an appropriate way to study the nature of the strong coupling regime of the impurity problem. This can be done by analyzing the stability of the point $h=\infty$. When $h \gg 1$, one can consider the spin-spin coupling in the spin chain as a small perturbation in a real space $\mathrm{RG}$ analysis. This procedure generates a new operator, which is not irrelevant, and therefore the fixed point $h=\infty$ proves to be unstable. Both limits, $h=0$ and $h=\infty$, are repulsive, and consequently there should be an attractive fixed point at an intermediate finite value of $h$. Since $h \propto u_{-}$, this analysis yields the existence of the repulsive $L_{\infty}$-line and the attractive $L_{f}$-line on the left part of the RG flow diagram depicted in Fig. 1.

1. A mapping of impurity scattering in TL liquids onto a spin- $1 / 2$ semi-infinite chain.

It is convenient to describe a point-like impurity scattering by a pair of chiral variables 18 


$$
\begin{aligned}
& \Theta_{e}(x)=\frac{1}{2 \sqrt{2}}[(\tilde{\phi}(x)+\tilde{\phi}(-x))-(\phi(x)-\phi(-x))], \\
& \Theta_{o}(x)=\frac{1}{2 \sqrt{2}}[(\tilde{\phi}(x)-\tilde{\phi}(-x))-(\phi(x)+\phi(-x))],
\end{aligned}
$$

that obey the commutation relations

$$
\begin{aligned}
{\left[\Theta_{e}(x), \Theta_{e}(y)\right]=} & {\left[\Theta_{o}(x), \Theta_{o}(y)\right]=-\frac{i}{4} \operatorname{sgn}(x), } \\
& {\left[\Theta_{e}(x), \Theta_{o}(y)\right]=-\frac{i}{4} }
\end{aligned}
$$

In terms of $\Theta_{e}, \Theta_{o}$ the Hamiltonian $H=H_{T L}+H_{s c}$, see Eqs. (9) and (13), can be rewritten in the form

$$
\begin{gathered}
H=H_{e}+H_{o} \\
H_{o}=\frac{v_{F}}{g} \int d x\left[\left(\frac{\partial \Theta_{o}}{\partial x}\right)^{2}-u_{-} \frac{g}{\pi \eta} \cos \left(\beta \sqrt{2} \Theta_{o}(x)\right) \delta(x)\right], \\
H_{e}=\frac{v_{F}}{g} \int d x\left[\left(\frac{\partial \Theta_{e}}{\partial x}\right)^{2}-u_{+} \frac{\beta g}{\sqrt{2} \pi} \frac{\partial \Theta_{e}(x)}{\partial x} \delta(x)\right] .
\end{gathered}
$$

Although $\Theta_{e}$ and $\Theta_{o}$ do not commute, the Hamiltonian $H$ is divided into even and odd parts, $H_{e}$ and $H_{o}$, respectively, because the even part contains only derivatives of $\Theta_{e}$. For small bare scattering amplitudes $\delta_{ \pm}$, and a not too strong electron-electron interaction, the linearization of the spectrum of the electrons used in the TL model is valid. The decoupling of forward and backward scattering holds under this approximation. For simplicity we omit the phase $\varphi_{u}$, and the $u_{a}$-term related to time reversal asymmetry, in $H_{o}$.

We now show that the odd part $H_{o}$ is effectively equivalent to the Hamiltonian of a semi-infinite spin-1/2 antiferromagnetic chain with anisotropy $\bar{\gamma}$ :

$H_{s}=\frac{J}{2} \sum_{n=0}^{\infty}\left(S_{n}^{+} S_{n+1}^{-}+S_{n}^{-} S_{n+1}^{+}\right)+\bar{\gamma} J \sum_{n=0}^{\infty}\left(S_{n}^{+} S_{n}^{-}-\frac{1}{2}\right)\left(S_{n+1}^{+} S_{n+1}^{-}-\frac{1}{2}\right)-h J\left(S_{0}^{-}+S_{0}^{+}\right)$.

A Hamiltonian of this type, with $\bar{\gamma}=0$, has been introduced by Guinea [21] for the description of a quantum particle interacting with a dissipative environment, at a particular value 
of the friction coefficient. It has also been used to discuss the transmission through barriers in TL liquids [5], for a given value of the electron-electron interaction $g=1 / 2$. Here we introduce the $\bar{\gamma}$-term in order not to be limited to a particular value of the electron-electron interaction. Note, that in $H_{s}$, unlike the standard tight binding model (see, e.g., Ref. [14]), the defect is located on a single site $j=0$, and therefore it has no internal structure. This is consistent with our purpose to study a point-like impurity.

To show the equivalence of $H_{o}$ and $H_{s}$, one should perform a sequence of transformations. After applying the inverse of the Jordan-Wigner transformation [22,23]

$$
S_{n}^{+}=c^{\dagger}(n) e^{i \pi \sum_{j=0}^{n-1} c_{j}^{\dagger} c_{j}}, S_{n}^{-}=e^{-i \pi \sum_{j=0}^{n-1} c_{j}^{\dagger} c_{j}} c(n)
$$

$H_{s}$ transforms into $H_{c}$, where

$$
H_{c}=\frac{J}{2} \sum_{j=0}^{\infty} c_{j}^{\dagger} c_{j+1}+h . c .+\bar{\gamma} J \sum_{j=0}^{\infty}\left(n_{j}-\frac{1}{2}\right)\left(n_{j+1}-\frac{1}{2}\right)-h J\left(c_{0}^{\dagger}+c_{0}\right) .
$$

In the absence of the local magnetic field $h$, the average of $S^{z}=\sum_{i} S_{i}^{z}$ over the ground state $|G\rangle$ of the spin chain is equal to zero. Since

$$
S^{z}=\sum_{j=0}^{N} c_{j}^{\dagger} c_{j}-N / 2=N_{F}-N / 2
$$

the sector $\left\langle G\left|S^{z}\right| G\right\rangle=0$ corresponds to half filling. The local operator $h J S_{0}^{x}$ cannot change the bulk properties of the chain. Therefore, it will be assumed that the fermion system $H_{c}$ of Eq.(A5) is at half filling. The continuum limit of $H_{c}$ (e.g., see Ref. [22]) corresponds to the effective Hamiltonian $H_{c}^{c o n t}=H_{0}+H_{\text {int }}+H_{h}$, where

$$
\begin{aligned}
& H_{0}=i v_{F} \int_{0}^{\infty} d x\left(L^{\dagger}(x) \partial_{x} L(x)-R^{\dagger}(x) \partial_{x} R(x)\right), \\
& H_{\text {int }}=v_{F} \bar{\gamma} \int_{0}^{\infty} d x\left(\bar{\rho}_{L}^{2}+\bar{\rho}_{R}{ }^{2}+4 \bar{\rho}_{R} \bar{\rho}_{L}\right)-2 \bar{\gamma} v_{F} \int_{0}^{\infty} d x\left[\left(R^{\dagger}(x) L(x)\right)^{2}+\left(L^{\dagger}(x) R(x)\right)^{2}\right], \\
& H_{h}=v_{F} \frac{1}{\sqrt{\eta}} h\left(R(x=0)+L(x=0)+R^{\dagger}(x=0)+L^{\dagger}(x=0)\right) .
\end{aligned}
$$

Here $v_{F}=J \eta$, where $\eta$ is the lattice spacing, and the operators $L$ and $R$ represent left and right movers on a semi-infinite line. The remnant of the discrete structure of the 
chain is the last term in $H_{\text {int }}$, which corresponds to the Umklapp processes at half filling 24,25]. This Umklapp term scales to zero for $|\bar{\gamma}|<1$, and also renormalizes the electronelectron interaction with a small momentum transfer. However, at small $\bar{\gamma}$ the latter effect is negligible, and for the low energy description one may substitute $H_{\text {int }}$ by the effective term

$$
H_{\text {int }}^{\prime}=v_{F} \bar{\gamma} \int_{0}^{\infty}\left(\bar{\rho}_{L}^{2}+\bar{\rho}_{R}^{2}+4 \bar{\rho}_{R} \bar{\rho}_{L}\right) d x
$$

The last step, which needs to be carried out in order to establish the equivalence between $H_{o}$ and $H_{s}$, is to unfold the semi-infinite line with left and right movers into a full line with a single chiral bosonic field. This yields:

$$
\begin{aligned}
H_{\text {chiral }}= & \pi v_{F} \int_{-\infty}^{\infty} d x\left[\rho_{c h}^{2}(x)+\bar{\gamma} / \pi\left(\rho_{c h}^{2}(x)+2 \rho_{c h}(x) \rho_{c h}(-x)\right)\right] \\
& -\frac{4 v_{F}}{\sqrt{2 \pi} \eta} h \cos \left(\sqrt{4 \pi} \Theta_{c h}(x=0)\right)
\end{aligned}
$$

where $d \Theta_{c h}(x) / d x=\sqrt{\pi} \rho_{c h}(x)$. Diagonalizing the quadratic part of the Hamiltonian, we find:

$$
\bar{H}_{\text {chiral }}=\frac{v_{F}}{g_{c h}} \int_{-\infty}^{\infty} d x\left[\pi \bar{\rho}_{c h}^{2}(x)-\frac{4 g_{c h}}{\sqrt{2 \pi} \eta} h \cos \left(\beta_{c h} \bar{\Theta}_{c h}(x)\right) \delta(x)\right],
$$

where $\beta_{c h}=\sqrt{4 \pi} \exp \chi, \chi=\frac{1}{2} \operatorname{arctanh}(2 \bar{\gamma} /(\pi+\bar{\gamma}))$, and $g_{c h} \approx(1+\bar{\gamma} / \pi)^{-1}$. Notice, the important role of the $\bar{\gamma}$-term - it modifies $\beta_{c h}$ inside the cosine term.

Thus, as a result of the sequence of transformations

$$
H_{s} \Longrightarrow H_{c} \Longrightarrow H_{c}^{c o n t} \Longrightarrow H_{\text {chiral }} \Longrightarrow \bar{H}_{\text {chiral }} \Longrightarrow H_{o}
$$

we obtain that the Hamiltonians $H_{s}$ and $H_{o}$ are equivalent when $\beta_{c h}=\sqrt{2} \beta$ and $h=$ $u_{-} / \sqrt{8 \pi}$. Due to the $\bar{\gamma}$-term, this equivalence is extended here to a finite interval of the electron-electron interaction.

\section{A scaling analysis of the spin chain model.}

We will use the equivalence of $H_{o}$ and $H_{s}$ to analyze the stability of the fixed line at $u_{-}=\infty$ and small $u_{+}$. A simple variant of the Nozières and Blandin approach [15] in their 
analysis of the two channel Kondo problem will be considered. Following this approach, we will assume that $h \gg 1$, and check whether the fixed point $h=\infty$ is a stable one.

In the presence of a strong magnetic field $h \gg 1$, the spin at site 0 is oriented along the direction opposite to the magnetic field. Its coupling to the nearest neighbor at the lattice site 1, can be treated as a perturbation. The reduced Hamiltonian that includes only sites 0 and 1 is given by

$$
H_{s}^{01}=\frac{J}{2}\left(S_{0}^{+} S_{1}^{-}+S_{0}^{-} S_{1}^{+}\right)+\bar{\gamma} J\left(S_{0}^{+} S_{0}^{-}-\frac{1}{2}\right)\left(S_{1}^{+} S_{1}^{-}-\frac{1}{2}\right)-h J\left(S_{0}^{-}+S_{0}^{+}\right) .
$$

After performing the permutation $x \rightarrow z, y \rightarrow x$, and $z \rightarrow y$ we obtain the Hamiltonian

$$
H_{s}^{01}=J S_{0}^{z} S_{1}^{z}+\frac{J}{4}(1-\bar{\gamma})\left[S_{0}^{+} S_{1}^{+}+S_{0}^{-} S_{1}^{-}\right]+\frac{J}{4}(1+\bar{\gamma})\left[S_{0}^{-} S_{1}^{+}+S_{0}^{+} S_{1}^{-}\right]-2 h J S_{0}^{z} .
$$

For $h \gg 1$ the spin at site 0 is in the state $|0 \uparrow\rangle$. Up to the first order in $J$ we have

$$
\left\langle 1 \downarrow\left|\left\langle 0 \uparrow\left|H_{s}^{01}\right| 0 \uparrow\right\rangle\right| 1 \downarrow\right\rangle=-J / 4, \quad\left\langle 1 \uparrow\left|\left\langle 0 \uparrow\left|H_{s}^{01}\right| 0 \uparrow\right\rangle\right| 1 \uparrow\right\rangle=J / 4 .
$$

This means that the spin at site 1 is under the action of an effective magnetic field $\tilde{h}=-1 / 4$. Under the assumption that $h \gg 1$, the higher orders in the perturbation theory give small corrections of the order of $h^{-1}$. As a result of this renormalization procedure step, we arrive at a problem equivalent to the initial one: a semi-infinite spin- $\frac{1}{2}$ Heisenberg chain, with a local magnetic field acting on the site at origin of the chain (now it will be site 1).

If one assumes that the discussed fixed point is such that the local magnetic field at the origin of the spin chain flows to infinity, then the renormalization procedure generates a relevant operator that makes this process nonconvergent. This is in contradiction with the initial assumption that $h=\infty$ is a stable fixed point. We have to conclude that $h=\infty$ is a repulsive fixed point. Note, that this conclusion holds for a finite interval of the parameter $|\bar{\gamma}| \lesssim 1$, because $\bar{\gamma}$ does not radically influence the effective magnetic field acting on site 1 . One can see this from the second order corrections to the energy of the anti-parallel and parallel spin configurations, that are $-J \frac{(1 \pm \bar{\gamma})^{2}}{32} h^{-1}$. 
It is not accidental that the present discussion resembles the analysis of the overscreened two channel Kondo problem [15]. Indeed, the spin chain model, in the absence of the $\bar{\gamma}$ term, is equivalent to the TL-impurity problem at a particular value of an electron-electron interaction parameter $g=1 / 2$ [21:5]. The latter problem can in turn be reduced to a resonant level model [26]. Moreover, the overscreened two channel Kondo model at a specific value of the longitudinal exchange coupling is equivalent to the same resonant level model [27]. Thus, the spin chain model and the Kondo model are equivalent at one point. On the other hand, it is well known that in the overscreened two channel Kondo problem the limit of infinite exchange interaction is unstable, and there is an anomalous fixed point at a finite coupling [15]. This property is preserved in the presence of the spin exchange anisotropy, which is irrelevant [28]. Since the spin chain model and the two channel Kondo model are equivalent at one point, it is natural that we have found that the point $h=\infty$ is repulsive. Since $h \propto u_{-}$, it follows from this analysis that the line $u_{-} \gg 1$ is a repulsive fixed line for the problem of impurity scattering in a TL liquid. The present treatment is not restricted to the special point of a TL liquid with $g=1 / 2$. This has been accomplished by the $\bar{\gamma} \neq 0$ term in the spin chain model. 


\section{REFERENCES}

$\dagger \quad$ Also at the Landau Institute for Theoretical Physics, Russia.

[1] G. D. Mahan, Many-Particle Physics, Second Edition (Plenum Press, New-York and London, 1990).

[2] D. C. Mattis, The many body problem (World Scientific Publishing Co., Singapure, 1993), chapter 4.

[3] X. G. Wen, Phys. Rev. B 44, 5708 (1991).

[4] D. C. Mattis, Phys. Rev. Lett. 32, 714 (1974).

[5] C. L. Kane and M. P. A. Fisher, Phys. Rev. B 46, 15233 (1992).

[6] K. A. Matveev, D. Yue, and L. I. Glazman, Phys. Rev. Lett. 71, 3351 (1993).

[7] A. Furusaki and N. Nagaosa, Phys. Rev. B 47, 4631 (1993).

[8] M. Ogata and H. Fukuyama, Phys. Rev. Lett. 73, 468 (1994).

[9] P. Fendley, A. W. W. Ludwig, and H. Saluer, Phys. Rev. Lett. 74, 3005 (1995).

[10] C. L. Kane and M. P. A. Fisher, Phys. Rev. Lett. 68, 1220 (1992).

[11] K. A. Matveev and L. I. Glazman, Phys. Rev. Lett. 70, 990 (1993).

[12] Y. Oreg and A. M. Finkel'stein, Phys. Rev. Lett. 76, 4230 (1996).

[13] Y. Oreg and A. M. Finkel'stein, Phys. Rev. B 53, 10928 (1996).

[14] S. Eggert and I. Affleck, Phys. Rev. B 46, 10866 (1992).

[15] P. Nozieres and A. Blandin, J. Physique 41, 193 (1980).

[16] Sometimes it is also called 'a weak link junction' or 'open boundary conditions'.

[17] C. L. Kane, K. A. Matveev, and L. I. Glazman, Phys. Rev. B 49, 2253 (1994). 
[18] I. Affleck and W. W. Ludwig, J. Phys. A: Math and Gen. 27, 5375 (1994).

[19] S. A. Bulgadaev, Teor. Matem. Fiz. 51, 424 (1982), Phys. Lett. A 86, 213 (1981).

[20] U. Weiss, Quantum Dissipative Systens (World Scientific, Singapore, New Jersey, London, Honk Kong, 1995).

[21] F. Guinea, Phys. Rev. B 32, 7518 (1985).

[22] E. Fradkin, in Field Theories of Condensed Matter Systems, Frontiers in Physics (Addison-Wesley, Redwood City, CA, 1991), Chap. 4.

[23] A. Luther and I. Peschel, Phys. Rev. B 12, 3908 (1975).

[24] F. D. M. Haldane, Phys. Rev. B 25, 4925 (1982).

[25] F. D. M. Haldane, Phys. Rev. Lett. 45, 1358 (1980).

[26] K. A. Matveev, Phys. Rev. B 51, 1743 (1995).

[27] V. J. Emery and S. Kivelson, Phys. Rev. B 46, 10812 (1992).

[28] I. Affleck, A. W. W. Ludwig, H. B. Pang, and D. L. Cox, Phys. Rev. B 45, 7918 (1992). 